\title{
Weak convergence and averaging for ODE
}

\author{
Lawrence C. Evans* and Te Zhang \\ Department of Mathematics \\ University of California, Berkeley
}

\begin{abstract}
This mostly expository paper shows how weak convergence methods provide simple, elegant proofs of (i) the stabilization of an inverted pendulum under fast vertical oscillations, (ii) the existence of particle traps induced by rapidly varying electric fields and (iii) the adiabatic invariance of $\int_{\Gamma} p d x$ for slowing varying planar Hamiltonian dynamics. Under an appropriate, but very restrictive, unique ergodicity assumption, the proof of (iii) extends also to many degrees of freedom.
\end{abstract}

For Juan Luis Vazquez on his 70th birthday.

\section{Introduction.}

The rigorous mathematical analysis of nonlinear differential equations depends primarily upon deriving estimates, but typically also upon using these estimates to justify limiting procedures of various sorts. For the latter, so-called weak convergence methods can be extremely valuable, as illustrated by many examples in the booklet [E].

This paper provides some more examples, concerning averaging effects for singularly perturbed nonlinear ODE. Section 2 shows how some simple "nonlinear resonance" effects (occurring when the weak limit of the product of two sequences of functions is not the product of the individual weak limits) appear for Kapitsa's inverted pendulum and its generalizations. Section 3 invokes the more sophisticated tools of Young measures to document the adiabatic invariance of the volume within constant energy surfaces for slowly changing Hamiltonian systems, provided an appropriate ergodic type condition holds. Our proofs are perhaps new, at least in the elegant versions we provide, and our presentation is largely expository.

We wish also to call attention to Bornemann's book [B], a very interesting discussion of weak convergence methods applied to singularly perturbed mechanical and quantum systems.

*Class of 1961 Collegium Chair. Supported in part by NSF Grant DMS-1301661.

(C) 2016. This manuscript version is made available under the Elsevier user license

http://www.elsevier.com/open-access/userlicense/1.0/ 
His primarily interest is explaining how increasingly singular potentials enforce holonomic constraints in the limit.

The results in Section 2 appear in somewhat different form in the second author's 2014 $\mathrm{PhD}$ thesis from UC Berkeley. We thank M. Zworski for explaining to us about ergodicity for Hamiltonian systems.

\section{$2 \quad$ Averaging and stability}

2.1 The inverted pendulum. The equation of motion for an inverted pendulum over a vertically oscillating pivot is

$$
\theta_{\epsilon}^{\prime \prime}-\left(a+\frac{b}{\epsilon} \cos \frac{t}{\epsilon}\right) \sin \theta_{\epsilon}=0
$$

where $\theta_{\epsilon}=\theta_{\epsilon}(t)$ denotes the angle from the vertical and $a:=\frac{g}{l}>0, l$ denoting the length. This is Kapitsa's pendulum: see for example Landau-Lifshitz [L-L, §30], Arnold [A, §25.E] and Levi [L1].

We provide a simple proof that solutions of (2.1) converge as $\epsilon \rightarrow 0$ to solutions of $\theta^{\prime \prime}+\frac{b^{2}}{4} \sin 2 \theta-a \sin \theta=0$. This ODE has the form $\theta_{t t}+F^{\prime}(\theta)=0$, for which the solution $\theta \equiv 0$ is stable provided $F^{\prime \prime}(0)=\frac{b^{2}}{2}-a>0$; that is, if and only if $|b| \geq \sqrt{2 a}$. This is the well-known stability condition for the inverted pendulum in the high frequency limit.

We turn now to a rigorous proof. Consider the following initial-value problem:

$$
\left\{\begin{aligned}
\theta_{\epsilon}^{\prime \prime} & =\left(a+\frac{b}{\epsilon} \cos \frac{t}{\epsilon}\right) \sin \theta_{\epsilon} \quad(t \geq 0) \\
\theta_{\epsilon}(0) & =\alpha \\
\theta_{\epsilon}^{\prime}(0) & =\beta
\end{aligned}\right.
$$

THEOREM 2.1. As $\epsilon \rightarrow 0, \theta_{\epsilon}$ converges uniformly on each finite time interval $[0, T]$ to the solution $\theta$ of

$$
\left\{\begin{aligned}
\theta^{\prime \prime} & =a \sin \theta-\frac{b^{2}}{4} \sin 2 \theta \\
\theta(0) & =\alpha \\
\theta^{\prime}(0) & =\beta
\end{aligned}\right.
$$

The main idea will be to rewrite the ODE (2.2) into the form

$$
\left(\theta_{\epsilon}^{\prime}-b \sin \frac{t}{\epsilon} \sin \theta_{\epsilon}\right)^{\prime}=a \sin \theta_{\epsilon}-b \sin \frac{t}{\epsilon} \cos \theta_{\epsilon} \theta_{\epsilon}^{\prime} .
$$

Proof. 1. First we show that for each $T>0$, we have the estimate

$$
\max _{0 \leq t \leq T}\left|\theta_{\epsilon}\right|,\left|\theta_{\epsilon}^{\prime}\right| \leq C_{T}
$$


for a constant $C_{T}>0$ that only depends on $T, \alpha$ and $\beta$. To confirm this, integrate (2.4), to find

$$
\left|\theta_{\epsilon}^{\prime}(t)\right| \leq C_{1}+C_{2} \int_{0}^{t}\left|\theta_{\epsilon}^{\prime}\right| d s
$$

for $0 \leq t \leq T$ and constants $C_{1}, C_{2} \geq 0$. According then to Gronwall's inequality, we have the estimate

$$
\left|\theta_{\epsilon}^{\prime}(t)\right| \leq C_{1}\left(1+C_{2} t e^{C_{1} t}\right) \leq C_{T}
$$

for each $0 \leq t \leq T$ and a constant $C_{T}>0$ that only depends on $T$.

2. Using (2.5), we can find a subsequence $\epsilon_{j} \rightarrow 0$ such that

$$
\begin{cases}\theta_{\epsilon_{j}} \rightarrow \theta & \text { uniformly on }[0, T] \\ \theta_{\epsilon_{j}}^{\prime} \rightarrow \theta^{\prime} & \text { weakly in } L^{2}(0, T)\end{cases}
$$

We next claim that

$$
\theta_{\epsilon_{j}}^{\prime} \sin \frac{t}{\epsilon_{j}} \rightarrow \frac{b}{2} \sin \theta \quad \text { weakly in } L^{2}(0, T)
$$

as $\epsilon \rightarrow 0$. To see this, observe that for all $\psi \in C^{\infty}([0, T])$ vanishing near $t=0, T$, we have

$$
\begin{aligned}
\int_{0}^{T} \psi \sin \frac{t}{\epsilon} \theta_{\epsilon}^{\prime} d t & =-\epsilon \int_{0}^{T} \psi\left(\cos \frac{t}{\epsilon}\right)^{\prime} \theta_{\epsilon}^{\prime} d t \\
& =\epsilon \int_{0}^{T} \psi^{\prime} \cos \frac{t}{\epsilon} \theta_{\epsilon}^{\prime} d t+\epsilon \int_{0}^{T} \psi \cos \frac{t}{\epsilon} \theta_{\epsilon}^{\prime \prime} d t \\
& =O(\epsilon)+\int_{0}^{T} \psi \cos \frac{t}{\epsilon}\left(\epsilon a+b \cos \frac{t}{\epsilon}\right) \sin \theta_{\epsilon} d t \\
& =O(\epsilon)+\int_{0}^{T} b \psi \cos ^{2} \frac{t}{\epsilon} \sin \theta_{\epsilon} d t \\
& \rightarrow \frac{b}{2} \int_{0}^{t} \sin \theta \psi d t
\end{aligned}
$$

as $\epsilon=\epsilon_{j} \rightarrow 0$, since $\cos ^{2} \frac{t}{\epsilon} \rightarrow \frac{1}{2}$. This proves (2.6).

3. Now integrate (2.4):

$$
\theta_{\epsilon}^{\prime}(t)-b \sin \frac{t}{\epsilon} \sin \theta_{\epsilon}(t)=\beta+\int_{0}^{t} a \sin \theta_{\epsilon}-b \sin \frac{s}{\epsilon} \cos \theta_{\epsilon} \theta_{\epsilon}^{\prime} d s
$$

for $0<t<T$. Let $\epsilon=\epsilon_{j} \rightarrow 0$ and pass to weak limits, recalling (2.6):

$$
\theta^{\prime}(t)=\beta+\int_{0}^{t} a \sin \theta-\frac{b^{2}}{2} \cos \theta \sin \theta d s=\beta+\int_{0}^{t} a \sin \theta-\frac{b^{2}}{4} \sin 2 \theta d s .
$$


The function $\theta$ is therefore smooth on $[0, T]$ and solves the ODE $\theta^{\prime \prime}=a \sin \theta-\frac{b^{2}}{4} \sin 2 \theta$, with $\theta^{\prime}(0)=\beta$. Since $\theta_{\epsilon_{j}} \rightarrow \theta$ locally uniformly, $\theta(0)=\alpha$ as well.

Since the initial value problem (2.3) has a unique solution $\theta$, we see that in fact the full sequence $\left\{\theta_{\epsilon}\right\}_{\epsilon>0}$ converges: $\theta_{\epsilon} \rightarrow \theta$ locally uniformly.

2.2 Generalization. We next generalize to the system of ODE

$$
\left\{\begin{aligned}
\mathbf{x}_{\epsilon}^{\prime \prime} & =\frac{1}{\epsilon} g\left(\frac{t}{\epsilon}\right) \mathbf{f}\left(\mathbf{x}_{\epsilon}\right) \\
\mathbf{x}_{\epsilon}(0) & =\alpha \\
\mathbf{x}_{\epsilon}^{\prime}(0) & =\beta
\end{aligned}\right.
$$

Here $\mathbf{x}_{\epsilon}=\mathbf{x}_{\epsilon}(t)=\left(x_{\epsilon}^{1}(t), \ldots, x_{\epsilon}^{n}(t)\right)$ and $\mathbf{f}: \mathbb{R}^{n} \rightarrow \mathbb{R}^{n}$ is a smooth function with

$$
\sup _{\mathbb{R}^{n}}|D \mathbf{f}|<\infty
$$

We assume $g: \mathbb{R} \rightarrow \mathbb{R}$ is continuous, 1-periodic, and

$$
\int_{0}^{1} g(t) d t=\int_{0}^{1} t g(t) d t=0 .
$$

Define $G(t):=\int_{0}^{t} g(s) d s$; then $G$ is 1 -periodic,

$$
G(0)=G(1)=0, \quad \int_{0}^{1} G d t=0
$$

Write

$$
<G^{2}>:=\int_{0}^{1} G^{2} d t
$$

THEOREM 2.2. As $\epsilon \rightarrow 0$, we have $\mathbf{x}_{\epsilon} \rightarrow \mathbf{x}$ uniformly on each finite time interval $[0, T]$, where $\mathbf{x}$ is the unique solution of

$$
\left\{\begin{aligned}
\mathbf{x}^{\prime \prime} & =-<G^{2}>D \mathbf{f}(\mathbf{x}) \mathbf{f}(\mathbf{x}) \\
\mathbf{x}(0) & =\alpha \\
\mathbf{x}^{\prime}(0) & =\beta
\end{aligned}\right.
$$

Remark. In the conservative case that

$$
\mathbf{f}=D \phi
$$


for a scalar potential function $\phi$, the limit dynamics read

$$
\mathbf{x}^{\prime \prime}=-D \psi(\mathbf{x})
$$

for the new potential function

$$
\psi:=\frac{<G^{2}>}{2}|D \phi|^{2} .
$$

We consequently have local stability near any nondegenerate critical point of $\phi$. As explained by M. Levi in [L2], this is the principle behind the "Paul trap" in physics.

Proof. 1. Rewrite the ODE (2.7) as

$$
\left(\mathbf{x}_{\epsilon}^{\prime}-G\left(\frac{t}{\epsilon}\right) \mathbf{f}\left(\mathbf{x}_{\epsilon}\right)\right)^{\prime}=-G\left(\frac{t}{\epsilon}\right) D \mathbf{f}\left(\mathbf{x}_{\epsilon}\right) \mathbf{x}_{\epsilon}^{\prime}
$$

Integrating and noting $|\mathbf{f}(z)| \leq C+C|z|$, we see that

$$
\left|\mathbf{x}_{\epsilon}^{\prime}(t)\right| \leq C+C\left|\mathbf{x}_{\epsilon}(t)\right|+C \int_{0}^{t}\left|\mathbf{x}_{\epsilon}^{\prime}(s)\right| d s
$$

for all $t \geq 0$. Since $\left|\mathbf{x}_{\epsilon}(t)\right| \leq C+\int_{0}^{t}\left|\mathbf{x}_{\epsilon}^{\prime}(s)\right| d s$, it follows that

$$
\left|\mathbf{x}_{\epsilon}^{\prime}(t)\right| \leq C+C \int_{0}^{t}\left|\mathbf{x}_{\epsilon}^{\prime}(s)\right| d s
$$

for appropriate constants $C$. Gronwall's inequality therefore implies that for each $T>0$, we have the estimate

$$
\max _{0 \leq t \leq T}\left|\mathbf{x}_{\epsilon}(t)\right|,\left|\mathbf{x}_{\epsilon}^{\prime}(t)\right| \leq C_{T}
$$

for a constant $C_{T}>0$ that only depends on $T, \alpha$ and $\beta$.

2. Hence for some sequence $\epsilon_{j} \rightarrow 0$,

$$
\mathbf{x}_{\epsilon_{j}} \rightarrow \mathbf{x} \quad \text { uniformly on }[0, T], \mathbf{x}_{\epsilon_{j}}^{\prime} \rightarrow \mathbf{x}^{\prime} \quad \text { weakly in } L^{2}\left(0, T ; \mathbb{R}^{n}\right) .
$$

We claim that

$$
G\left(\frac{t}{\epsilon_{j}}\right) \mathbf{x}_{\epsilon_{j}}^{\prime} \rightarrow<G^{2}>\mathbf{f}(\mathbf{x}) \quad \text { weakly in } L^{2}\left(0, T ; \mathbb{R}^{n}\right) .
$$

To see this, select any $\psi \in C^{\infty}([0, T])$ vanishing near $t=0, T$, and observe

$$
\int_{0}^{T} \psi G\left(\frac{t}{\epsilon}\right) \mathbf{x}_{\epsilon}^{\prime} d t=\epsilon \int_{0}^{T} \psi \Gamma\left(\frac{t}{\epsilon}\right)^{\prime} \mathbf{x}_{\epsilon}^{\prime} d t
$$


where $\Gamma(t):=\int_{0}^{t} G(s) d s$. Since $\int_{0}^{1} G d t=0$, the function $\Gamma$ is 1 -periodic. Therefore

$$
\begin{aligned}
\int_{0}^{T} \psi G\left(\frac{t}{\epsilon}\right) \mathbf{x}_{\epsilon}^{\prime} d t & =O(\epsilon)-\epsilon \int_{0}^{T} \psi \Gamma\left(\frac{t}{\epsilon}\right) \mathbf{x}_{\epsilon}^{\prime \prime} d t \\
& =O(\epsilon)-\int_{0}^{T} \psi \Gamma\left(\frac{t}{\epsilon}\right) g\left(\frac{t}{\epsilon}\right) \mathbf{f}\left(\mathbf{x}_{\epsilon}\right) d t \\
& \rightarrow-<\Gamma g>\int_{0}^{T} \mathbf{f}(\mathbf{x}) \psi d t
\end{aligned}
$$

as $\epsilon=\epsilon_{j} \rightarrow 0$, where

$$
<\Gamma g>:=\int_{0}^{1} \Gamma g d t=\int_{0}^{1} \Gamma G^{\prime} d t=-\int_{0}^{1} \Gamma^{\prime} G d t=-<G^{2}>
$$

This proves (2.11).

3. Now integrate (2.10):

$$
\mathbf{x}_{\epsilon}^{\prime}(t)-G\left(\frac{t}{\epsilon}\right) \mathbf{f}\left(\mathbf{x}_{\epsilon}(t)\right)=\beta-\int_{0}^{t} G\left(\frac{s}{\epsilon}\right) D \mathbf{f}\left(\mathbf{x}_{\epsilon}\right) \mathbf{x}_{\epsilon}^{\prime} d s
$$

and use (2.11) to pass to weak limits as $\epsilon=\epsilon_{j} \rightarrow 0$ :

$$
\mathbf{x}^{\prime}(t)=\beta-<G^{2}>\int_{0}^{t} D \mathbf{f}(\mathbf{x}) \mathbf{f}(\mathbf{x}) d s
$$

Notice that $G\left(\frac{t}{\epsilon}\right) \rightarrow \int_{0}^{1} G d t=0$. It follows that $\mathbf{x}$ is smooth when $\mathbf{f}$ is, and solves the ODE and second initial condition in (2.9). The first initial condition is also clear, since $\mathbf{x}_{\epsilon_{j}} \rightarrow \mathbf{x}$ locally uniformly.

As (2.9) has a unique solution, in fact the full sequence converges as $\epsilon \rightarrow 0$.

\section{Averaging and adiabatic invariance}

3.1 Slowly varying Hamiltonians. Let $H: \mathbb{R}^{n} \times \mathbb{R}^{n} \times \mathbb{R} \rightarrow \mathbb{R}, H=H(p, x, t)$, be a smooth, time-dependent family of Hamiltonians. Fix $T>0$ and consider then the system of ODE

$$
\left\{\begin{array}{l}
\dot{\mathbf{x}}^{\epsilon}=D_{p} H\left(\mathbf{p}^{\epsilon}, \mathbf{x}^{\epsilon}, \epsilon \tau\right) \\
\dot{\mathbf{p}}^{\epsilon}=-D_{x} H\left(\mathbf{p}^{\epsilon}, \mathbf{x}^{\epsilon}, \epsilon \tau\right)
\end{array} \quad\left(0 \leq \tau \leq \frac{T}{\epsilon}\right)\right.
$$

where $\cdot=\frac{d}{d \tau}$, with given initial conditions $\mathbf{x}^{\epsilon}(0)=x_{0}, \mathbf{p}^{\epsilon}(0)=p_{0}$. 
In these dynamics the Hamiltonians are varying slowly, but for a long time. An adiabatic invariant for (3.1) is some quantity involving the trajectory $(\mathbf{p}, \mathbf{x})$ that is approximately constant for times $0 \leq \tau \leq \frac{T}{\epsilon}$. Consult Arnold [A] and Arnold-Kozlov-Neishtadt [A-K-N] for the theory of adiabatic invariants and Crawford $[\mathrm{C}]$ for many examples.

For $n=1$ degrees of freedom, it is standard wisdom in physics that the action

$$
\Phi=\int_{\Gamma} p d x
$$

is an adiabatic invariant, where the integral is over a complete cycle $\Gamma$ of the motion. Arnold and others have given a rigorous interpretation and derivation of this assertion. We provide next a proof using weak convergence tricks, valid even for more degrees of freedom if the Hamiltonian dynamics are appropriately uniquely ergodic on each energy surface. We do not use action-angle variables.

3.2 Rescaling, weak convergence. We hereafter assume that $H \geq 0$,

$$
\lim _{(p, x) \rightarrow \infty} H=\infty \quad \text { uniformly on }[0, T]
$$

and

$$
\left|H_{t}\right| \leq C(1+H) \quad \text { on } \mathbb{R}^{n} \times \mathbb{R}^{n} \times[0, T]
$$

for some constant $C$.

We now rescale in time, setting

$$
t:=\epsilon \tau, \mathbf{x}_{\epsilon}(t):=\mathbf{x}^{\epsilon}\left(\frac{t}{\epsilon}\right), \mathbf{p}_{\epsilon}(t):=\mathbf{p}^{\epsilon}\left(\frac{t}{\epsilon}\right)
$$

Then

$$
\left\{\begin{array}{l}
\mathbf{x}_{\epsilon}^{\prime}=\frac{1}{\epsilon} D_{p} H\left(\mathbf{p}_{\epsilon}, \mathbf{x}_{\epsilon}, t\right) \\
\mathbf{p}_{\epsilon}^{\prime}=-\frac{1}{\epsilon} D_{x} H\left(\mathbf{p}_{\epsilon}, \mathbf{x}_{\epsilon}, t\right)
\end{array} \quad(0 \leq t \leq T),\right.
$$

where ${ }^{\prime}=\frac{d}{d t}$. Equivalently, we write

$$
\mathbf{z}_{\epsilon}^{\prime}=\frac{1}{\epsilon} J D_{z} H\left(\mathbf{z}_{\epsilon}, t\right) \quad(0 \leq t \leq T)
$$

for $\mathbf{z}_{\epsilon}:=\left(\mathbf{p}_{\epsilon}, \mathbf{x}_{\epsilon}\right), D_{z} H=\left(D_{p} H, D_{x} H\right)$, and $J:=\left(\begin{array}{cc}O & -I \\ I & O\end{array}\right)$.

The energy at time $t$ is

$$
e_{\epsilon}:=H\left(\mathbf{p}_{\epsilon}, \mathbf{x}_{\epsilon}, t\right)
$$




\section{LEMMA 3.1.}

(i) We have

$$
e_{\epsilon}^{\prime}=H_{t}\left(\mathbf{p}_{\epsilon}, \mathbf{x}_{\epsilon}, t\right) \quad(0 \leq t \leq T) .
$$

(ii) There exists a constant $C$ such that

$$
\sup _{0 \leq t \leq T}\left|\mathbf{p}_{\epsilon}\right|,\left|\mathbf{x}_{\epsilon}\right| \leq C
$$

for each $0<\epsilon \leq 1$.

(iii) There exists a sequence $\epsilon_{j} \rightarrow 0$ and a continuous function e such that

$$
e_{\epsilon_{j}} \rightarrow e \text { uniformly on }[0, T]
$$

Proof. Calculating (3.7) is immediate from (3.5). It follows then from hypothesis (3.4) that

$$
e_{\epsilon}^{\prime} \leq C+C e_{\epsilon}
$$

whence Gronwall's inequality implies $\sup _{0 \leq t \leq T} e_{\epsilon} \leq C$. The estimate (3.8) is now a consequence of the coercivity assumption (3.3). Finally, (3.7) and (3.8) imply (3.9) for an appropriate subsequence.

Notation. We write for for $0 \leq t \leq T$

$$
\begin{aligned}
\Gamma(t) & :=\{(p, x) \mid H(p, x, t)=e(t)\}, \Gamma_{\epsilon}(t):=\left\{(p, x) \mid H(p, x, t)=e_{\epsilon}(t)\right\} \\
\Delta(t) & :=\{(p, x) \mid H(p, x, t) \leq e(t)\}, \Delta_{\epsilon}(t):=\left\{(p, x) \mid H(p, x, t) \leq e_{\epsilon}(t)\right\} .
\end{aligned}
$$

We hereafter assume also that

$$
\left|D_{z} H\right| \geq \gamma>0 \text { on } \Gamma(t), \Gamma_{\epsilon}(t) \quad(0 \leq t \leq T) .
$$

Consequently, $\Gamma(t), \Gamma_{\epsilon}(t)$ are smooth hypersurfaces, with outward unit normal $\nu:=\frac{D_{z} H}{\left|D_{z} H\right|}$; and we suppose as well that $\Gamma(t), \Gamma_{\epsilon}(t)$ are connected. Then for each continuous function $F$,

$$
\int_{\Gamma_{\epsilon_{j}}(t)} F d \mathcal{H}^{2 n-1} \rightarrow \int_{\Gamma(t)} F d \mathcal{H}^{2 n-1}
$$

uniformly on $[0, T]$, where $\mathcal{H}^{2 n-1}$ denotes Hausdorff measure.

LEMMA 3.2. Passing if necessary to a further subsequence and reindexing, we have for almost every time $0 \leq t \leq T$ a Borel probability measure $\sigma(t)$ on $\mathbb{R}^{n} \times \mathbb{R}^{n}$ such that

$$
\operatorname{spt} \sigma(t) \subseteq \Gamma(t),
$$




$$
\{H(\cdot, t), \sigma(t)\}=\operatorname{div}\left(J D_{z} H(\cdot, t) \sigma(t)\right)=0
$$

weakly in $\mathbb{R}^{n} \times \mathbb{R}^{n}$, and

$$
F\left(\mathbf{p}_{\epsilon_{j}}, \mathbf{x}_{\epsilon_{j}}, t\right) \rightarrow \bar{F}:=\int_{\Gamma(t)} F(p, x, t) d \sigma(t)
$$

for each continuous function $F$.

Proof. 1. The existence of a (possibly further) subsequence $\epsilon_{j} \rightarrow 0$ and Young measures $\sigma(t)$ such that

$$
F\left(\mathbf{p}_{\epsilon_{j}}, \mathbf{x}_{\epsilon_{j}}, t\right) \rightarrow \int_{\mathbb{R}^{n} \times \mathbb{R}^{n}} F(p, x, t) d \sigma(t)
$$

for continuous functions $F$ follows as in Tartar [T] or [E]. The assertion (3.11) follows from (3.10), since $\left(\mathbf{p}_{\epsilon_{j}}, \mathbf{x}_{\epsilon_{j}}\right) \in \Gamma_{\epsilon_{j}}(t)$. Consequently (3.13) holds.

2. To prove (3.12), let $\phi=\phi(p, x, t)$ be smooth, with compact support in $\mathbb{R}^{n} \times \mathbb{R}^{n} \times(0, T)$. Put $F=J D H \cdot D \phi$. Then, since $\phi$ vanishes at $t=0, T$, we have

$$
\begin{aligned}
O(\epsilon) & =-\epsilon \int_{0}^{T} \phi_{t}\left(\mathbf{z}_{\epsilon}, t\right) d t \\
& =\epsilon \int_{0}^{T} \phi\left(\mathbf{z}_{\epsilon}, t\right)^{\prime}-\phi_{t}\left(\mathbf{z}_{\epsilon}, t\right) d t \\
& =\int_{0}^{T} J D_{z} H \cdot D \phi\left(\mathbf{z}_{\epsilon}, t\right) d t .
\end{aligned}
$$

Therefore (3.13) implies

$$
0=\lim _{\epsilon_{j} \rightarrow 0} \int_{0}^{T} J D_{z} H \cdot D \phi\left(\mathbf{z}_{\epsilon_{j}}, t\right) d t=\int_{0}^{T} \int_{\mathbb{R}^{n} \times \mathbb{R}^{n}} J D_{z} H \cdot D \phi d \sigma(t) d t .
$$

The validity of this identity for each $\phi$ is the weak formulation of (3.12).

We introduce next Liouville measure on $\Gamma(t)$, defined for Borel sets $E$ by the rule

$$
\mu(t)(E):=\frac{1}{Z(t)} \int_{\Gamma(t) \cap E} \frac{1}{\left|D_{z} H\right|} d \mathcal{H}^{2 n-1}
$$

and normalized by $Z(t):=\int_{\Gamma(t)} \frac{1}{\left|D_{z} H\right|} d \mathcal{H}^{2 n-1}$.

LEMMA 3.3. For each time $0 \leq t \leq T$ we have

$$
\{H(\cdot, t), \mu(t)\}=0
$$

weakly in $\mathbb{R}^{n} \times \mathbb{R}^{n}$. 
Proof. Let $\phi=\phi(p, x)$ be smooth, with compact support. Then

$$
\begin{aligned}
\int_{\mathbb{R}^{n} \times \mathbb{R}^{n}} J D_{z} H \cdot D \phi d \mu(t) & =\frac{1}{Z(t)} \int_{\Gamma(t)} \nu \cdot J^{T} D \phi d \mathcal{H}^{2 n-1} \\
& =\frac{1}{Z(t)} \int_{\Delta(t)} \operatorname{div}\left(J^{T} D \phi\right) d z \\
& =0
\end{aligned}
$$

since $J^{T}$ is antisymmetric. Therefore $\{H(\cdot, t), \mu(t)\}=\operatorname{div}\left(J D_{z} H(\cdot, t) \mu(t)\right)=0$ in the weak sense.

Now define

$$
\Phi(t):=|\Delta(t)|, \Phi_{\epsilon}(t):=\left|\Delta_{\epsilon}(t)\right| \quad(0 \leq t \leq T)
$$

to be the $2 n$-dimensional volumes of $\Delta(t), \Delta_{\epsilon}(t)$.

THEOREM 3.4. Assume for each time $0 \leq t \leq T$ that the Liouville measure $\mu(t)$ is the unique Borel probability measure $\mu$ supported on $\Gamma(t)$ solving

$$
\{H(\cdot, t), \mu\}=0
$$

weakly in $\mathbb{R}^{n} \times \mathbb{R}^{n}$.

Then

$$
\Phi_{\epsilon}^{\prime} \rightarrow 0
$$

as $\epsilon \rightarrow 0$, and consequently

$$
\Phi \text { is constant on }[0, T] \text {. }
$$

Remark. The hypothesis that (3.18) has a unique solution supported in $\Gamma(t)$ is called unique ergodicity and is extremely strong for $n>1$.

A heuristic derivation of a special case of this assertion, but without the uniqueness hypothesis for (3.18), is in Appendix D of Campisi-Kobe [C-K], who discuss also the interpretation of $S=k_{B} \log \Phi$ as the microcanonical Gibbs entropy of the classical Hamiltonian system. See also Dunkel-Hilbert [D-H] for more discussion; they credit Hertz with the observation that $\Phi$, and thus $S$, are adiabatic invariants.

Bornemann $[\mathrm{B}]$ uses weak convergence methods to derive quantum adiabatic theorems. 
Proof. The hypersurface $\Gamma_{\epsilon}(t)$ is the 0 level set of the function $W^{\epsilon}=W^{\epsilon}(z, t):=H(z, t)-$ $e_{\epsilon}(t)$, whose outward normal velocity is therefore $-\frac{W_{t}^{\epsilon}}{\left|D_{z} W^{\epsilon}\right|}$. Thus

$$
\begin{aligned}
\Phi_{\epsilon}(t)^{\prime} & =-\int_{\Gamma_{\epsilon}(t)} \frac{W_{t}^{\epsilon}}{\left|D_{z} W^{\epsilon}\right|} d \mathcal{H}^{2 n-1} \\
& =\int_{\Gamma_{\epsilon}(t)} \frac{e_{\epsilon}^{\prime}(t)-H_{t}(p, x, t)}{\left|D_{z} H\right|} d \mathcal{H}^{2 n-1} \\
& =\int_{\Gamma_{\epsilon}(t)} \frac{H_{t}\left(\mathbf{p}_{\epsilon}, \mathbf{x}_{\epsilon}, t\right)-H_{t}(p, x, t)}{\left|D_{z} H\right|} d \mathcal{H}^{2 n-1}
\end{aligned}
$$

according to (3.7). But owing to (3.11)-(3.13) and the assumed uniqueness of probability measures solving (3.18), it follows that the Young measure $\sigma(t)$ equals the normalized Liouville measure for a.e. time. Thus

$$
H_{t}\left(\mathbf{p}_{\epsilon_{j}}, \mathbf{x}_{\epsilon_{j}}, t\right) \rightarrow \frac{1}{Z(t)} \int_{\Gamma(t)} \frac{H_{t}}{\left|D_{z} H\right|} d \mathcal{H}^{2 n-1} .
$$

Since $\int_{\Gamma_{\epsilon_{j}}(t)} \frac{H_{t}}{\left|D_{z} H\right|} d \mathcal{H}^{2 n-1} \rightarrow \int_{\Gamma(t)} \frac{H_{t}}{\left|D_{z} H\right|} d \mathcal{H}^{2 n-1}$ and $\int_{\Gamma_{\epsilon_{j}}(t)} \frac{1}{\left|D_{z} H\right|} d \mathcal{H}^{2 n-1} \rightarrow \int_{\Gamma(t)} \frac{1}{\left|D_{z} H\right|} d \mathcal{H}^{2 n-1}=$ $Z(t)$ uniformly on $[0, T]$, we have $\Phi_{\epsilon_{j}}^{\prime} \rightarrow 0$.

This assertion holds as well for an appropriate subsequence of any given sequence $\epsilon_{k} \rightarrow 0$, and consequently $\Phi_{\epsilon}^{\prime} \rightarrow 0$.

3.3 One degree of freedom. For one degree of freedom, the unique ergodicity hypothesis holds automatically, since the level sets of $H(\cdot, t)$ are diffeomorphic to circles:

THEOREM 3.5. If $n=1$, then

$$
\Phi_{\epsilon}^{\prime} \rightarrow 0
$$

as $\epsilon \rightarrow 0$, and consequently

$$
\Phi \text { is constant on }[0, T] \text {. }
$$

Since Green's Theorem implies $\int_{\Gamma(t)} p d x= \pm \Phi(t)$ (depending upon the orientation), we recover the classical assertion about adiabatic invariance for one degree of freedom.

Proof. Suppose $\mu$ is a Borel probability measure supported in $\Gamma=\Gamma(t)$ and satisfying

$$
\{H, \mu\}=0 .
$$

for $H=H(\cdot, t)$. Then

$$
\int_{\Gamma} J \nu \cdot D \phi\left|D_{z} H\right| d \mu=0
$$

for each smooth $\phi$. 
Let $\{\mathbf{w}(s)=(p(s), x(s)) \mid 0 \leq s \leq L\}$ be a unit speed parameterization of $\Gamma$, oriented so that $\mathbf{w}^{\prime}=\tau:=J \nu$ and ${ }^{\prime}=\frac{d}{d s}$. Then

$$
0=\int_{\Gamma} J \nu \cdot D \phi\left|D_{z} H\right| d \mu=\int_{0}^{L} \psi^{\prime} d \tilde{\nu}
$$

where $\tilde{\nu}$ is the pushforward of $\nu=\left|D_{z} H\right| \mu$ under $\mathbf{w}^{-1}$ and $\psi=\phi(\mathbf{w})$. The foregoing identity for each $\psi$ satisfying $\psi(0)=\psi(L)$ implies that $\tilde{\nu}$ is a constant multiple of one-dimensional Lebesgue measure. This shows that $\nu=\mathcal{H}^{1}$ on $\Gamma$, times an appropriate normalizing constant. Hence $\mu$ is the Liouville measure.

\section{References}

[A] V.I. Arnold, Mathematical Methods of Classical Mechanics, trans by K. Vogtmann and A. Weinstein, Springer, 1978

[A-K-N] V.I. Arnold, V.V. Kozlov and A.I. Neishtadt, Mathematical Aspects of Classical and Celestial Mechanics, trans by A. Iacob, Vol 3 of Encyclopedia of Mathematical Sciences, Springer, 1988

[B] F. Bornemann, Homogenization in Time for Singularly Perturbed Mechanical Systems, Lecture Notes in Mathematics 1687, Springer, 1998

[C-K] M. Campisi and D.H. Kobe, Derivation of the Boltzmann principle, American J Phys. 78 (2010), 608-615.

[C] F. Crawford, Elementary examples of adiabatic invariance, American J Phys. 58 (1990), 337-344.

[D-H] J. Dunkel and S. Hilbert, Consistent thermostatics forbids negative temperatures, Nature Phys. 10 (2014), 67-72.

[E] L.C. Evans, Weak Convergence Methods for Nonlinear Partial Differential Equations, American Math Society, CBMS \#74, American Mathematical Society, 1990. Third printing, 2002.

[L-L] L.D. Landau and E.M. Lifshitz, Mechanics, 3rd ed, trans by J.B. Sykes and J.S. Bell, Vol 1 of Course in Theoretical Physics, Pergammon, 1976

[L1] M. Levi, Geometry of Kapitsa's potentials, Nonlinearity 11 (1998), 1365-1368.

[L2] M. Levi, Geometry and physics of averaging with applications, Phys. D 132 (1999), 150-164. 
[T] L. Tartar, Compensated compactness and applications to partial differential equations, pages 136-212 in Nonlinear Analysis and Mechanics, Heriot-Watt Symposium, Vol. IV, Pitman, 1979. 